\title{
Fine structure of profiles of weak diffuse interstellar bands ${ }^{\star}$
}

\author{
G. Galazutdinov ${ }^{1,2,3}$, W. Stachowska ${ }^{1}$, F. Musaev ${ }^{1,2,3}$, C. Moutou ${ }^{4}$, G. Lo Curto ${ }^{5}$, and J. Krełowski ${ }^{1}$ \\ 1 Center for Astronomy, Nicholas Copernicus University, Gagarina 11, 87-100 Toruń, Poland \\ e-mail: jacek@astri.uni.torun.pl; wiolka@astri.uni.torun.pl \\ 2 Special Astrophysical Observatory, Nizhnij Arkhyz 369167, Russia \\ e-mail: gala@sao.ru; faig@sao.ru \\ 3 Isaac Newton Institute of Chile, SAO Branch \\ ${ }^{4}$ Laboratorie d'Astrophysique de Marseille, Traverse du Siphon, Les trois Lucs, BP 8, 13376 Marseille Cedex 12, France \\ e-mail: Claire.Moutou@oamp.fr \\ 5 European Southern Observatory, Alonso De Cordova 3107, Vitacura, Santiago, Chile \\ e-mail: glocurto@eso.org
}

Received 17 July 2002 / Accepted 2 September 2002

\begin{abstract}
We present a very high resolution $(R=120000-220000)$ analysis of profiles of selected diffuse interstellar bands (DIBs): 4964, 4980, 4985, $5850 \AA$. The $4980 \AA$ feature is a very weak one, yet some substructures are proved to be present, as well as within the profiles of another stronger ones where this phenomenon was found several years ago. The presence of substructures inside DIB profiles supports their molecular origin.
\end{abstract}

Key words. ISM: clouds, molecules

\section{Introduction}

The first observations of diffuse interstellar bands (DIBs) were made on photographic plates, usually with too low resolution and signal-to-noise ratio to allow the detection of possible internal structures of their profiles. However, as early as 1975, Herbig first suggested that DIB profiles may differ from single Gaussians. With the coming of the era of CCD+echelle spectroscopy, the observational possibilities grew substantially. Herbig \& Soderblom (1982) demonstrated convincingly that the profiles of diffuse interstellar bands, as well as those of interstellar atomic lines, can be modified by Doppler splitting. The same was shown later by Westerlund \& Krełowski (1988) where only the broadening of some DIB profiles caused by the Doppler effect was presented. Heavily reddened stars, i.e. the objects in which DIBs were discovered, are usually obscured by several clouds and the Doppler splitting is very likely to modify their profile shapes and - to a certain extent - their wavelengths.

Westerlund \& Krełowski (1989) were the first to analyze the profiles of five DIBs along sightlines where atomic (NaI) lines do not show any Doppler splitting. The latter analysis showed that all DIB profiles are non-Gaussian and that some

Send offprint requests to: J. Krełowski, e-mail: jacek@astri.uni.torun.pl

* Based on data collected at the ESO $3.6 \mathrm{~m}$ telescope operated on La Silla Observatory, Chile and 2-m telescope of the Terskol Observatory, Russia. substructures exist inside the relatively narrow DIBs (5797 and 6379). These observations initiated investigations of the intrinsic profiles of DIBs, i.e. profiles not modified by the Doppler effect - the only observational results which may be directly compared to those of laboratory gas phase experiments. Such profiles can be observed only in individual clouds i.e. in the case of low to moderate reddening (single clouds of very high reddening are not common). Westerlund \& Krełowski (1989), after their analysis of intrinsic DIB profiles, concluded that their widths are constant, independently of the environment where they originated. The only broadening possible is because of the Doppler splitting along sightlines towards distant stars. This conclusion was disproved soon after by Porceddu et al. (1992). They found physically broadened (but also very weak) diffuse band profiles in the Orion OB1 association. The latter result was confirmed recently by Krełowski \& Greenberg (1999).

Any attempt to identify some of the DIBs must involve an analysis of their profiles, especially their contours which are likely to be specific to a particular species. Also their rest wavelengths should be known with reasonable precision. These requirements lead to the use of high resolution spectra acquired with a high $S / N$ ratio. Rest wavelengths can be determined using radial velocities measured from profiles of interstellar atomic lines recorded in the spectra of the same targets. It is, however, not proved that the diffuse band carriers have the same spatial distribution and motion as the atoms and diatomic molecules observed along the same lines of sight 
(Krełowski \& Greenberg 1999). The cases where ALL the above mentioned features share the same radial velocity seem to be most certain as sources of velocity reference frames.

Krełowski \& Schmidt (1997) proved that some substructures are very likely to be present inside the profiles of almost all reasonably strong and rather narrow diffuse bands. This result confirmed that of Sarre et al. (1995) where the analysis was restricted to only a few DIBs and targets. The profiles showing internal structure are very likely to be molecular bands, originating in large molecules with low rotational constants which, together with turbulent motions of interstellar gas, make any further resolution of their rotational profiles impossible. The observed substructures are very narrow and shallow which makes their detection difficult. The very broad DIBs (i.e. $4430 \AA$ Å) do not show any internal structure (Snow 2002).

The highest resolution DIB profiles are those of Sarre et al. (1995) and Kerr et al. (1998). The Ultra High Resolution Facility allowed us to see some very significant details of the 5797 and 6614 profiles. Rotational resolution routinely achieved in the laboratory is not likely in astronomical spectra because of Doppler broadening, but the rotational profile should be preserved. Data acquired by Kerr et al. (1998) with resolving power $R=600000$ on $\lambda 5797$ DIB resemble the rotational contour of an unresolved $P, Q$ and $R$-branch (Fig. 1). The profiles demonstrate some variability i.e. they apparently depend on physical parameters of interstellar clouds. This phenomenon was shown in the case of 6196, 6379 and 6614 DIBs in the recent papers by Walker et al. (2001).

The profiles of recently discovered, weak DIBs have not been determined until now with high resolution and high $S / N$ ratio. Provided that they originate in the same molecules which also carry the strong features, they should include similar substructures inside their profiles. However, the task of resolving very weak features into substructures is even more difficult than that of doing the same with reasonably strong DIBs.

\section{Observational data}

We used spectra with resolution $R=120000$ acquired with the aid of the Coudé Echelle Spectrometer (Musaev et al. 1999) fed by the $2 \mathrm{~m}$ telescope of the Observatory on top of Terskol Peak (Northern Caucasia) to search for possible substructures inside the DIB profiles. High resolution allows profile analysis of the observed features. The targets (see Table 1 in Galazutdinov et al. 2002ba) were chosen using the profiles of atomic interstellar lines obtained along the lines of sight towards them. We have chosen only those objects where the Doppler splitting is barely seen in very narrow atomic lines. Profiles of DIBs are much broader and thus we may consider them as free of the Doppler effect. The objects are sufficiently bright to allow recording of high resolution, high $S / N$ spectra.

The spectrometer, working with the Wright Instruments CCD $1242 \times 1152$ matrix (pixel size $22.5 \mu \mathrm{m} \times 22.5 \mu \mathrm{m}$ ) camera, covers the range $\sim 3500 \AA-\sim 10100 \AA$ in three exposures with a resolution of $R=120000$. Any exposure covers approximately one third of the above-mentioned range. The available resolution makes elimination of telluric features much more

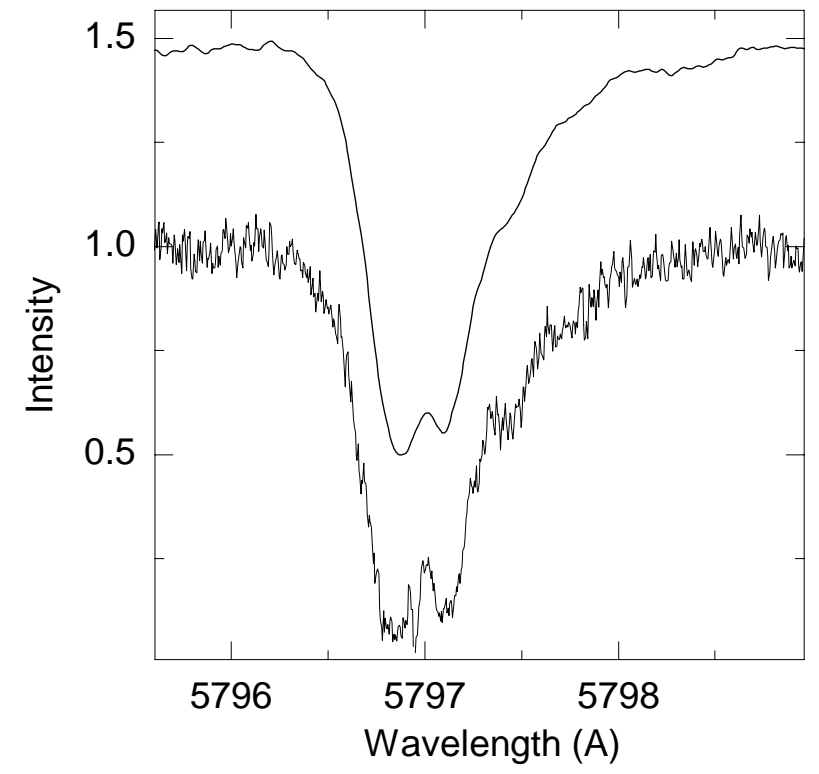

Fig. 1. Comparison of the profiles of DIB 5797 acquired with aid of the Coudé-echelle spectrograph MAESTRO (Musaev et al. 1999) fed with the $2 \mathrm{~m}$ telescope (top) and Ultra High Resolution Facility at AngloAustralian Observatory (kindly provided by P. Sarre) at the bottom. Resolving powers are 120000 and 600000 respectively. Both profiles are normalized to 1 for clarity. It clearly shows that the only difference is the very narrow structure in the center of DIB.

reliable than any data previously used for this purpose. The precise elimination of telluric contamination makes the measurement of wavelengths and intensities of the interstellar features reliable as well as improving the possible detection of substructures.

To search for possible fine structure of weak diffuse bands we have selected the spectral region already described by Galazutdinov et al. (2001) i.e. centered around the possible $C_{5}$ feature at $4975 \AA$. The region contains two well-known, not very strong DIBs: 4964 and 4985, possibly with some structure inside (Galazutdinov et al. 2001); some additional, weak interstellar features have been discovered only very recently, together with the above-mentioned possible $C_{5}$ feature.

The selected spectral region was also observed at ESO with the aid of the CES (Coude Echelle Spectrograph) fed by the fibre link with the Cassegrain focus of the $3.6 \mathrm{~m}$ telescope at La Silla Observatory. All the stars (see Table 1) have been observed with the highest resolving power, $R=220000$, using the Very Long Camera. The instrument is equipped with an image slicer which splits the starlight into a dozen wellilluminated slices. The detector is an EEV $2 \mathrm{~K} \times 4 \mathrm{~K} \mathrm{CCD}$ (pixel size $15 \times 15 \mu \mathrm{m}$ ) with $80 \%$ quantum efficiency in the domain of interest. The presence of well-known DIBs with precisely determined rest wavelengths (Galazutdinov et al. 2000) in the recorded wavelength range allows us to determine the precise rest wavelengths of the newly discovered weak features.

Our reduction of the spectra was done using the DECH code (Galazutdinov 1992). This program allows flatfield division, bias/background subtraction, one-dimensional spectrum extraction from the 2-dimensional images, correction for diffuse light, spectrum addition, excision of cosmic ray 
Table 1. The stars observed at ESO with $R=220000$. HD numbers, spectral and luminousity classes, $V$ magnitudes, reddening, rotational velocities are given. The remaining columns represent equivalent width measurements (in $m \AA$ ) of the DIBs with given wavelength. The signalto-noise ratio is given in parentheses.

\begin{tabular}{rlrrrrrr}
\hline \hline HD & SpL & $V$ & $E_{B-V}$ & $v \times \sin i$ & 4964 & 4980 & 4985 \\
\hline 144217 & B0.5V & 2.62 & 0.17 & 130 & $4.5 \pm 0.5(1400)$ & - & $1.8 \pm 0.2(2000)$ \\
147165 & B1III & 2.91 & 0.32 & 53 & $5.4 \pm 0.5(1500)$ & - & $2.8 \pm 0.4(1700)$ \\
148184 & B2IVpe & 4.42 & 0.44 & 134 & $23.1 \pm 0.7(1000)$ & $6.6 \pm 0.6(1100)$ & $8.3 \pm 0.5(1200)$ \\
149757 & O9.5V & 2.58 & 0.30 & 379 & $10.3 \pm 0.5(1200)$ & $10.5 \pm 0.5(1500)$ & $3.2 \pm 0.3(1900)$ \\
179406 & B3V & 5.36 & 0.31 & 150 & $22.0 \pm 1.0(700)$ & $6.6 \pm 0.8(800)$ & $9.0 \pm 0.7(800)$ \\
\hline
\end{tabular}

features, etc. The DECH code also allows location of a fiducial continuum, measurements of the line equivalent widths, line positions and shifts, etc. The flat-fielding procedure for high-resolution image-sliced CES spectra of very wide aperture (more than 350 pixels) is a very crucial point, especially when searching for very weak spectral features. To make sure that all of them are real (i.e. of astrophysical origin) we have also reduced the original images with the aid of well-known packages: IRAF and MIDAS and compared them. Below we discuss only the features which are seen in the spectra irrespective of the reduction package used.

\section{Results}

To illustrate the possibilities of the $R=120000$ Terskol spectra we have used the well-known 5797 DIB. Its profile has been observed in extremely high resolution by Kerr et al. (1998) and thus it is known to include several substructures likely to be different branches of a molecular band. We have averaged 10 spectra of two reddened stars: HD 23180 and HD 24398, members of the Per OB2 association. The spectra of clouds obscuring these two neighbouring stars are very similar, in particular the 5797/5780 strength ratio is almost identical in both objects. The 5797 DIB is relatively strong when observed in both these targets (Megier et al. 2001). The average of the 10 spectra of these stars achieved an $S / N$ ratio $\sim 1200$ around the 5797 DIB. In Fig. 1 a comparison of this average with the published 5797 profile, observed by Kerr et al. (1998) in the spectrum of HD 149757 characterized by the resolution $R=600000$ is shown. It is evident that we can trace in $R=120000$ all the spectral features visible in the extreme resolution, except the very narrow dip which probably is blended with the neighbouring broader feature.

The only reasonably strong diffuse band which has not been observed yet in high resolution is situated near $5850 \AA$. The band has already been found to be not exactly of Gaussian profile e.g. by Krełowski \& Schmidt (1997), but it was not possible to resolve possible substructures in the McDonald spectra of $R=60000$. Having the resolution twice as high one can try to reconsider the 5850 profile. We have averaged 15 spectra of HD 23180, HD 24398 and HD 24912 to build the profile of this feature. The final result is shown in Fig. 2. The profile should be free of stellar line contamination. As demonstrated by Krełowski \& Schmidt (1997), 5850 can only be blended with a reasonably weak FeIII line. Our Fig. 2 shows the neighbouring FeIII line which should be stronger than that possibly present inside the 5850 profile (see the arrow). It is

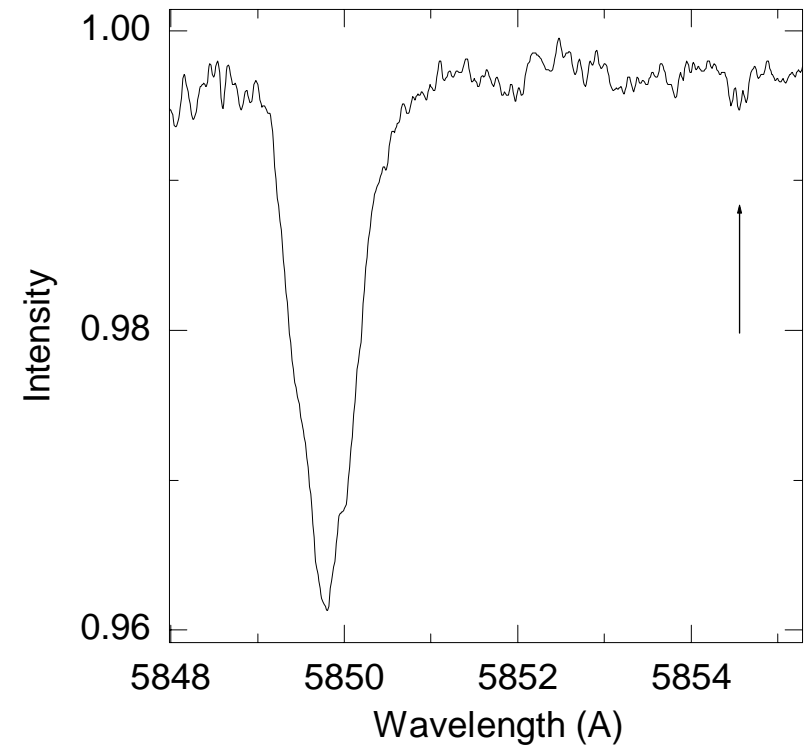

Fig. 2. DIB $5850 \AA$ profile $(R=120000)$ (see text).

evident that this line is very weak and thus the possible contamination of the DIB is most likely completely negligible. The DIB profile seems to be slightly asymmetrical and has weak substructure in the red wing.

Other investigated DIBs are in the vicinity of the possible $C_{5}$ feature (Galazutdinov et al. 2002b) - around $4975 \AA$. This spectral range was observed at ESO as well as at Terskol observatories. Every spectrum, both from ESO and the averaged from Terskol, clearly shows two well known diffuse bands $4964 \AA$ and $4985 \AA$. They have been mentioned by Galazutdinov et al. (2001) as possibly composed of substructures. Moreover one can trace a few additional weak features of apparently interstellar origin (Galazutdinov et al. 2001) in the close vicinity of the above mentioned DIBs. They cannot be identified as stellar lines because such features are absent in synthetic spectra of hot stars. They cannot be definitively explained by instrumental effects because the spectra were obtained in two different places (Terskol and ESO), with the data collected by different devices so the lines which repeat in both spectra cannot be instrumental structures. They also cannot be interpreted as atmospheric because in the discussed spectral range $\sim 4957-\sim 4992 \AA$ there are no known telluric lines. Weak, unidentified stellar lines also have to be excluded, because the HD 148184, HD 149757 and HD 179406 stars are fast rotators (Table 1). Weak stellar lines in fast rotating 


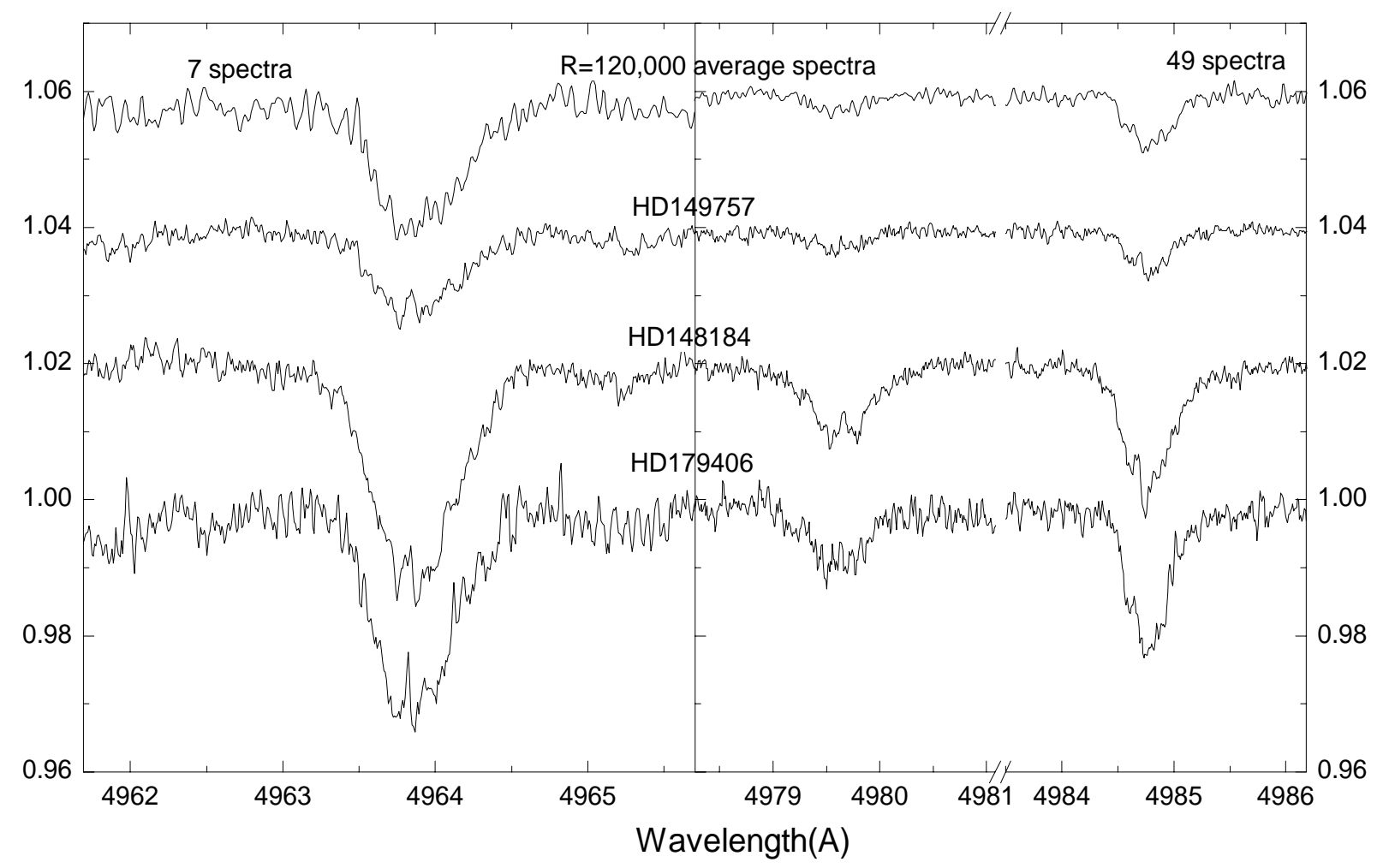

Fig. 3. DIBs 4964, 4980, $4985 \AA$ profiles. Top - average $R=120000$ Terskol spectrum. The lower (ESO) spectra are of $R=220000$ (see text).

objects would not be noticeable because of their strong broadening and flattening, so such structures would be hardly distinguishable from the continuum. The averaging of the spectra further weakens these structures because all the spectra were shifted so as to bring the interstellar features to their rest wavelengths. The amount of such a shift depends on the radial velocity of the interstellar cloud, and is different for each line of sight and so for each star. Therefore the shift places a particular stellar structure at a different position in each spectrum, and thus the averaging of the spectra causes an additional flattening of such structures. In this case several narrow and very weak features, discovered in the average spectrum by Galazutdinov et al. (2001) must be of interstellar origin. It is to be emphasized that our observations are restricted to interstellar clouds of reasonably low optical depths. The molecular abundances can easily be higher towards heavily reddened stars. However, such objects are usually too faint to be observed in high resolution with high $S / N$ ratio.

Studies of the line profiles are possible only when a single cloud of interstellar matter is considered. The main criterion which allows us to assume that there is more than one cloud between an observer and the star is the shape of the profiles of narrow atomic lines of interstellar origin. In the case of one cloud these should be symmetric and unaffected by Doppler splitting. An additional criterion is the reddening of the star situated beyond the cloud. The value of the reddening increases with the amount of matter. Therefore strongly reddened stars are more likely to be shining through several clouds which probably have different radial velocities relative to an observer. Every interstellar feature in the spectrum of such objects is the superimposed profile of the same line originating in each cloud.
This effect is especially noticeable in the case of the narrow atomic lines. Therefore, the objects listed in Table 1 were chosen to have (a) relatively small reddening of the stars and (b) no Doppler splitting of the KI line in their spectra.

The strongest structures in all the spectra from Fig. 3 are the two known diffuse interstellar lines at wavelengths $4963.90 \AA$ and $4984.81 \AA$. The 4964 DIB is characterized by an asymmetric profile with an extended wing in the longer wavelength range. At the bottom of the line one can trace two clearly visible substructures. This line has a very similar profile shape to that of the 5797 DIB which is presented in Fig. 1. The 4985 DIB is also asymmetric and the shorter wavelength side is steeper. The dominant component inside the profile is situated in the central part of the line and probably the two much weaker substructures appear on both sides of this profile.

For the first time we can see some evident substructures inside profiles of such weak DIBs. The substructures can be traced in both the Terskol spectra, being an average of 49 individual exposures of 17 targets, and in individual ESO spectra; this proves their existence beyond a doubt. Apparently, the presence of substructures inside DIB profiles is their common property irrespective of the intensity. Perhaps the similarity of high resolution profiles of certain DIBs may be an additional argument in favour of their common origin - together with their constant strength ratio (Weselak et al. 2001). It seems that a resolution above $R=100000$ is necessary to resolve substructure pattern in most of diffuse bands (see for comparison the $R=220000$ DIB profiles in the paper by Galazutdinov et al. 2002b). One can also argue that some asymmetry is a common property of DIB profiles. In all our plots this feature is clearly seen. 
In both Terskol and ESO spectra two clearly visible structures at wavelengths $4969.03 \AA$ and $4979.62 \AA$ are observed. They have been found by Galazutdinov et al. (2001) and marked as "new" because they are not listed in any of the published atlases of interstellar bands (see e.g. Weselak et al. 2001; Galazutdinov et al. 2001). The 4969 DIB is a very weak structure and therefore it is difficult to describe the details of the profile. Nonetheless, this line has a similar shape to the 4985 DIB, which was discussed earlier in this work, with one, dominant, centrally situated substructure. The 4980 DIB is of similar shape to the 4964 line, with two clear substructures within the bottom of profile. The asymmetry of the profile is noticeable, though this time the left, shorter-wavelength side is extended. In our ESO-CES spectra the latter feature clearly splits into two substructures. Apparently this kind of profile is very common among diffuse interstellar bands. The "sigma" type targets (HD 147165 and HD 144217) also observed at ESO show the above DIBs detectably, but so weak (the usual property of narrow features in "sigma" objects) that any analysis of the profiles is not possible.

The paper clearly proves that the presence of substructures in DIB profiles is their common property which can be useful for discovering their origin by comparing astrophysical data with laboratory gas phase spectra of (for example) carbon chains or PAHs (Salama et al. 1999; Motylewski et al. 2000). A high resolution and high signal-to-noise ratio of the astrophysical data are absolutely necessary conditions to perform this task. We consider a resolving power of $\sim 120000$ and higher as good enough while signal-to-noise ratio should be not lower than $\sim 1200-1500$, especially in the case of numerous weak diffuse interstellar bands.

Acknowledgements. The authors want to express their gratitude to the staff of the ESO and Terskol Observatories for the technical help during the observations. JK acknowledges the financial support of the
French-Polish project JUMELAGE. GAG wants to express his thanks to Russian Foundation for Basic Research for financial support under the grant No. 02-02-174423

\section{References}

Galazutdinov, G. A. 1992, Prep. Spets. Astrof. Obs., No. 92

Galazutdinov, G. A., Musaev, F. A., Krełowski, J., \& Walker, G. A. H. 2000, PASP, 112, 648

Galazutdinov, G. A., Musaev, F. A., \& Krełowski, J. 2001, MNRAS, 325,1332

Galazutdinov, G. A., Pȩtlewski, A., Musaev, F. A., et al. 2002a, A\&A, submitted

Galazutdinov, G. A., Moutou, C., Musaev, F., \& Krelowski, J. 2002b, A\&A, 384, 215

Herbig, G. H. 1975, ApJ, 196, 129

Herbig, G. H., \& Soderblom, D. R. 1982, ApJ, 252, 610

Kerr, T. H., Hibbins, R. E., Miles, J. R., et al. 1996, MNRAS, 283, 105

Krełowski, J., \& Greenberg, J. M. 1999, A\&A, 346, 199

Krełowski, J., \& Schmidt, M. 1997, ApJ, 477, 209

Megier, A., Aiello, S., Barsella, B., Casu, S., \& Krełowski, J. 2001, MNRAS, 326, 1095

Motylewski, T., Linnartz, H., Vaizert, O., et al. 2000, ApJ, 531, 312

Musaev, F. A., Galazutdinov, G. A., Sergeev, A. V., Karpov, N. V., \& Podyachev, Yu. V. 1999, Kinematika i Fizika Nebesnyh Tel, 15(3)

Porceddu, I., Benvenuti, P., \& Krełowski, J. 1992, A\&A, 260, 391

Salama, F., Galazutdinov, G. A., Krełowski, J., Allamandola, L. J., \& Musaev, F. A. 1999, ApJ, 526, 265

Sarre, P. J., Miles, J. R., Kerr, T. H., et al. 1995, MNRAS, 277, 41

Snow, T. P. 2002, ApJ, 567, 407

Walker, G. A. H., Webster, A. S., Bohlender, D. A., \& Krełowski, J. 2001, ApJ, 561, 272

Weselak, T., Fulara, J., Schmidt, M. R., \& Krełowski, J. 2001, A\&A, 377, 677

Westerlund, B. E., \& Krełowski, J. 1988, A\&A, 203, 134

Westerlund, B. E., \& Krełowski, J. 1989, A\&A, 218, 216 